\section{Gravidez na adolescência: perfil sócio- demográfico e comportamental de uma população da periferia de São Paulo, Brasil}

\author{
Teenage pregnancy: behavioral and socio- \\ demographic profile of an urban \\ Brazilian population
}

\footnotetext{
${ }^{1}$ Departamento de Psiquiatria, Universidade Federal de São Paulo, São Paulo, Brasil. 2 Institute of Psychiatry, King's College London, London, U.K.

3 Departamento de Pediatria Universidade Federal de São Paulo, São Paulo, Brasil.

Correspondência E. Chalem Unidade de Pesquisa em Álcool e Drogas, Departamento de Psiquiatria, Universidade Federal de São Paulo. Rua Marques do Paraná 567, apto. 42A, São Paulo, SP 05086-010, Brasil. elisachalem@uol.com.br
}

\begin{abstract}
To identify the socio-demographic behavioral profile of low-income pregnant teenagers, 1,000 adolescents admitted to a Brazilian public maternity hospital from July 24, 2001, to November 27, 2002, were interviewed. Socio-demographic and behavioral variables were assessed through a questionnaire. Over the 492 days of the study, 24.3\% of admissions were adolescents (930 for childbirth and 70 for miscarriage). Mean maternal age was 17 years. Most teenagers (72.9\%) lived near the hospital. 930 (93\%) belonged to socioeconomic classes $C, D$, and E. School dropout was identified in $67.3 \%$ of the total. $80.1 \%$ of the subjects were giving birth for the first time. $81.2 \%$ had not planned the pregnancy, and $23.8 \%$ had been using some contraceptive method. $67.4 \%$ had vaginal deliveries. Some $13.3 \%$ of the newborns were premature and $15.9 \%$ had low birth weight. 17.3\% of these adolescent mothers reported smoking during pregnancy, with $2.8 \%$ reporting alcohol and $1.7 \%$ illicit drugs. Teenage pregnancy is a complex phenomenon associated with various economic, educational, and behavioral factors. The study provides importance references for public policies to prevent teenage pregnancy.
\end{abstract}

Pregnancy in Adolescence; Adolescent; Social Class; Demography; Sexual Behavior

\author{
Elisa Chalem 1 \\ Sandro Sendin Mitsuhiro ${ }^{1}$ \\ Cleusa P. Ferri 2 \\ Marina Carvalho Moraes Barros 3 \\ Ruth Guinsburg 3 \\ Ronaldo Laranjeira 1
}

\section{Introdução}

Nas últimas décadas, a gestação na adolescência tem sido considerada um importante assunto de saúde pública, em virtude da prevalência com que esse fenômeno vem ocorrendo ao redor do mundo 1,2,3,4,5,6. A chamada epidemia da maternidade na adolescência só foi reconhecida por volta de 1970, quando as taxas de fecundidade nesta faixa etária já começavam a cair nos Estados Unidos 2 e em outros países do primeiro mundo.

No entanto, no quinto relatório anual do State of the World's Mothers, publicado em 2004 (http://www.savethechildren.org.uk) com dados coletados entre 1995 e 2002, Mayor destacou que 13 milhões de nascimentos (um décimo de todos os nascimentos do mundo) são de mulheres com menos de vinte anos e que mais de $90 \%$ destes nascimentos ocorrem nos países em desenvolvimento, onde a proporção de parturientes com menos de vinte anos varia de $8 \%$ no leste da Ásia até $55 \%$ na África 7 . O relatório alertou que a gravidez e o parto foram a principal causa de morte em mulheres de 15 a 19 anos nos países em desenvolvimento.

Vários estudos relatam como esse fenômeno se comporta em diferentes populações ao redor do mundo. Henshaw 8 observou que os maiores índices de gestação na adolescência recaíam preferencialmente sobre a parcela negra da população (duas a três vezes maior do que entre as 
brancas), na qual predomina o nível sócio-econômico baixo. Bennett et al. ${ }^{9}$ verificaram que a ocorrência de gravidez dos 15 aos 19 anos de idade é maior na zona rural do que nas áreas metropolitanas, onde, de uma forma geral, há mais acesso à educação e à informação. Singh ${ }^{10}$, de maneira semelhante, concluiu, em seu artigo, que níveis educacionais mais altos estão associados a menores índices de gestação na adolescência. Barnet et al. 11 relataram que gravidez na adolescência estava associada com o aumento na taxa de evasão escolar e que isso aumentaria a probabilidade de persistirem as diferenças econômicas e sociais.

Sobre os países desenvolvidos, diversos autores referem uma tendência de queda na proporção de gravidez na adolescência a partir dos anos 80. Arias et al. 12 relataram que, nos Estados Unidos, essa taxa caiu 31\% desde 1991. Creatas 13, num estudo em 11 países europeus, também observou a tendência de essa taxa se manter estável ou até diminuir. Na Alemanha, por exemplo, que tem a maior taxa de gravidez na adolescência da Europa, o índice caiu de 21\% em 1987 para 13\% em 1989.

No Brasil, segundo o Ministério da Saúde 14, cresceu a proporção da participação da gravidez entre 15 e 19 anos nos índices de fecundidade, paralelamente à diminuição da proporção das demais faixas etárias. Além disso, dados do SUS indicam que a porcentagem da faixa etária dos 10 aos 19 anos no total dos partos nos hospitais conveniados chegou a 26,5\% em 1997 contra 22,34\% em 1993. Os estudos de Gama et al. 15 e Sabroza et al. 16, no Rio de Janeiro, Ribeiro et al. 17, em Ribeirão Preto, e Simões et al. 18, em São Luís do Maranhão, destacam a alta e crescente taxa de gestações na adolescência, principalmente entre as mais jovens, apresentando particularidades de acordo com a região e a população estudada. Simões et al. 18 destacaram ainda a associação entre gestação na adolescência e prematuridade quando se controlam variáveis confundidoras, como fatores sócio-econômicos e reprodutivos.

Recentemente, na literatura médica, sociológica e na mídia, discutiu-se sobre a associação entre adolescência e problemas decorrentes tanto de fatores de natureza biológica, como da autonomia relativa e ambígua que os jovens desfrutam na família e na sociedade. Esse fenômeno surge em sociedades modernas e torna-se acentuado em processos de rápida urbanização 19. Nesse contexto, a Fundação Seade criou o índice de vulnerabilidade juvenil (IVJ), que inclui a proporção de mães adolescentes, e classificou os 96 distritos do Município de São Paulo.

O presente estudo tem como objetivo descrever as condições sócio-demográficas e com- portamentais, associadas temporalmente com a gestação na adolescência, numa região de periferia da cidade de São Paulo com um alto índice de vulnerabilidade juvenil.

\section{Metodologia}

Trata-se de um estudo de corte transversal descritivo, com coleta prospectiva dos dados, realizado no Hospital Municipal Maternidade-Escola Dr. Mário de Moraes Altenfelder Silva. Localizado na Zona Norte da cidade de São Paulo, é considerado hospital de nível terciário, de referência para as gestações de alto risco. A abrangência inclui as zonas Noroeste (sete distritos) e Nordeste (11 distritos), principalmente os distritos de Brasilândia e Cachoeirinha, e, desde a década de 80, em torno de $25 \%$ dos partos ali realizados são de adolescentes, sendo o hospital a primeira opção para o parto das adolescentes de toda a região. Está localizado a, aproximadamente, $8 \mathrm{~km}$ de distância do centro da cidade.

Cachoeirinha conta com 147.649 habitantes, dos quais $10,21 \%$ (15.075) são pessoas na faixa de 15 a 19 anos 19. A população nessa região é basicamente constituída por indivíduos de baixa renda, que não têm acesso aos serviços privados de saúde.

Este estudo faz parte do projeto temático Uso de Drogas por Gestantes Adolescentes, financiado pela Fundação de Amparo à Pesquisa do Estado de São Paulo (FAPESP - processo 00/10.293-5).

\section{Casuística}

Foram incluídas no estudo as adolescentes grávidas, consideradas aquelas com idades entre 10 e 20 anos incompletos (critério da Organização Mundial da Saúde) admitidas no hospital para parturição ou curetagem pós-abortamento. A inclusão se deu após a obtenção do consentimento livre e esclarecido por escrito. Não foram utilizados critérios de exclusão.

Este artigo é uma análise secundária, por isso os cálculos amostrais foram baseados no objetivo principal do projeto temático, que foi estudar a prevalência do uso de drogas e os fatores de risco para seu uso durante a gravidez. Tendo em vista a prevalência de drogas na ordem de $20 \%$ 20 e considerando-se um erro amostral de $2,5 \%$ a $3 \%$, estimou-se a necessidade de inclusão de 680 a 980 binômios mãe-recém-nascidos no estudo.

\section{Instrumentos}

As participantes foram entrevistadas nas enfermarias por entrevistadores independentes, sem qual- 
quer vínculo com a maternidade. Eram psicólogos treinados e com experiência prévia em pesquisa, contratados especificamente para esta tarefa.

O questionário foi elaborado tendo como base o Perinatal Needs Assessment (PNA) 21, instrumento utilizado em estudo realizado na Califórnia, Estados Unidos, envolvendo 1.147 gestantes, com o objetivo de analisar em que medida a rede social afeta a vida das mulheres grávidas. O PNA foi traduzido e adaptado à realidade brasileira $\mathrm{e}$ a particularidades da nossa população. Foram consideradas as variáveis referentes a dados:

- de identificação: idade, procedência e bairro de moradia, estado civil;

- de inserção social: escolaridade e situação profissional;

- de nível sócio-econômico: renda familiar; forma de sustento; condições de moradia e a classificação de classe econômica da Associação Nacional de Estudos e Pesquisa (ANEP) 22, baseada no nível educacional do chefe de família, no número de eletrodomésticos na residência e na renda familiar. A ANEP classifica os indivíduos em cinco categorias diferentes, de A (classe mais alta) a E (classe mais baixa);

- da gestação: planejamento; uso de anticoncepcionais; referência de ter realizado ou não pré-natal; local do pré-natal; média de consultas referidas e falta ao pré-natal;

- sobre o pai da criança e outros filhos;

- sobre a vida sexual: início da atividade sexual; informações sobre o número de parceiros sexuais que teve; estabilidade da relação que originou a gravidez; conhecimento sobre doenças sexualmente transmissíveis e utilização de preservativo durante as relações sexuais;

- sobre situações de violência a que já fora submetida;

- sobre o uso e abuso de substâncias tóxicas durante a gestação;

- sobre resultados da gestação: tipo de parto; idade gestacional e peso do recém-nascido.

\section{Aspectos éticos}

Este projeto foi aprovado pelos Comitês de Ética e Pesquisa do Hospital Municipal MaternidadeEscola Dr. Mario de Moraes Altenfelder Silva e da Universidade Federal de São Paulo (UNIFESP).

As participantes foram abordadas de 4 a 48 horas após a recuperação do parto ou da curetagem. Foram esclarecidas e orientadas a respeito do estudo pelos entrevistadores do projeto. Perguntava-se à paciente se concordaria ou não em assinar o Termo de Consentimento Livre e Esclarecido somente depois de totalmente dissipadas suas dúvidas. Em caso positivo, os entrevistadores aplicavam o questionário na enfermaria.

\section{Análise estatística}

O banco de dados foi constituído no programa Epi Info, versão 6.04 (Centers for Disease Control and Prevention, Atlanta, Estados Unidos). Para a análise dos dados, utilizou-se o pacote SPSS (SPSS Inc., Chicago, Estados Unidos). Foram elaboradas tabelas descritivas, utilizando-se as freqüências em números absolutos e percentuais. Algumas características das adolescentes de 15 anos ou menos foram comparadas com as das demais. O teste qui-quadrado foi usado para estabelecer a significância das diferenças.

\section{Resultados}

No período de julho de 2001 a novembro de 2002 (492 dias consecutivos), ocorreram 4.108 internações no centro obstétrico do hospital para parturição ou curetagem pós-abortamento, das quais $1.002(24,4 \%)$ eram adolescentes. Em duas destas não se conseguiu aplicar o questionário, sendo incluídas no estudo mil adolescentes. Todas concordaram em participar da pesquisa.

Das mil adolescentes, 70 (7\%) foram admitidas para curetagem e 930 (93\%) para parturição, sendo os conceptos 10 (1\%) óbitos fetais e 928 nascidos vivos (8 gemelares).

A média de idade das participantes foi de 17,0 anos (DP = 1,5), variando de 11 a 19 anos; $17 \%$ tinham até 15 anos. Como pode ser observado na Tabela 1, a grande maioria $(77,5 \%)$ era natural de São Paulo e as demais estavam na cidade há $6,9$ anos (DP $=5,5)$ em média, variando de 1 a 19 anos. A maior parte $(72,9 \%)$ das adolescentes morava em bairros adjacentes ao hospital. Quanto à estrutura familiar, apenas 7,2\% eram casadas legalmente, mas $62,7 \%$ referiam viver com um companheiro. Do total, $42,3 \%$ viviam exclusivamente com o companheiro e/ou filhos constituindo um núcleo familiar independente, ao passo que as demais $(57,7 \%)$ continuavam morando também com outros familiares $(15,1 \%$ com outros filhos, $42,9 \%$ com a mãe, $25,8 \%$ com os pais, $43 \%$ com irmãos). Conviviam na mesma casa em média três pessoas; em 22,6\% dos casos, mais de cinco pessoas.

Em relação à classe econômica, segundo a classificação da ANEP 22, 88,2\% das participantes pertenciam às classes $\mathrm{C}$ e D, sendo que $68 \%$ referiam renda familiar mensal de até quatro salários mínimos. A principal fonte de sustento provinha do companheiro e/ou pais da adolescente.

No que se refere à inserção social, a média de anos freqüentados de escola referidos foi de oito anos (DP $=2,3$ ), variando de 0 a 12 anos. Considerando-se a média de idade encontrada (17 anos), 
Características sócio-demográficas de adolescentes grávidas internadas numa maternidade municipal de São Paulo, Brasil.

\begin{tabular}{|c|c|c|}
\hline Características & $\mathrm{n}$ & $\%$ \\
\hline \multicolumn{3}{|l|}{ Procedência $(n=998)$} \\
\hline São Paulo & 773 & 77,5 \\
\hline Nordeste/Norte & 171 & 17,1 \\
\hline Sudeste/Sul & 39 & 3,9 \\
\hline Centro-Oeste & 12 & 1,2 \\
\hline Estrangeiro & 3 & 0,3 \\
\hline \multicolumn{3}{|l|}{ Bairro $(n=993)$} \\
\hline Brasilândia/Cachoeirinha & 556 & 56,2 \\
\hline Casa Verde/Freguesia do O/Limão & 168 & 16,9 \\
\hline Outros da Zona Norte & 208 & 20,8 \\
\hline Outros & 61 & 6,1 \\
\hline \multicolumn{3}{|l|}{ Estado civil $(n=1.000)$} \\
\hline Em união & 627 & 62,7 \\
\hline Sem união & 364 & 36,4 \\
\hline Viúva/Separada & 9 & 0,9 \\
\hline \multicolumn{3}{|l|}{ Tipo de moradia $(n=994)$} \\
\hline Casa & 859 & 86,5 \\
\hline Apartamento & 50 & 5,0 \\
\hline Cômodo/Barraco & 80 & 8,0 \\
\hline Outros & 5 & 0,5 \\
\hline \multicolumn{3}{|l|}{ Vínculo da moradia $(n=993)$} \\
\hline Própria & 657 & 66,2 \\
\hline Alugada & 274 & 27,6 \\
\hline Outros & 62 & 6,2 \\
\hline \multicolumn{3}{|l|}{ Classe econômica * $(n=995)$} \\
\hline A & 2 & 0,2 \\
\hline B & 64 & 6,4 \\
\hline C & 395 & 39,8 \\
\hline $\mathrm{D}$ & 487 & 48,9 \\
\hline E & 47 & 4,7 \\
\hline \multicolumn{3}{|c|}{ Renda familiar mensal em salários mínimos ** $(n=894)$} \\
\hline$\leq 1$ & 28 & 3,1 \\
\hline $1-4$ & 580 & 64,9 \\
\hline$>5$ & 286 & 32,0 \\
\hline
\end{tabular}

* Associação Nacional de Empresas de Pesquisa 22

** 1 salário mínimo $=\mathrm{R} \$ 180,00$ (2001)

a defasagem entre o esperado e o efetivamente cursado foi de 2,4 anos. Destaca-se que 67,3\% das adolescentes não estavam mais estudando no momento da entrevista; $60,2 \%$ associavam o abandono da escola com a gravidez e $65,4 \%$ haviam abandonado durante o ano letivo. Relataram ter recebido algum treinamento profissional específico $88(8,8 \%)$ adolescentes. Do total de participantes, 9,7\% referiam estar trabalhando. Como pode ser observado na Figura 1, o número de adolescentes que referiram estar estudando ou estar inseridas no mercado de trabalho varia de acordo com a idade considerada. Este núme- ro era sempre muito baixo comparado ao total de adolescentes de cada faixa. Do geral, observase que, independentemente da faixa de idade, a maioria das adolescentes não estudava nem trabalhava na ocasião da entrevista.

Em relação ao comportamento sexual, a média de idade de início de atividade sexual foi de 15 anos (DP $=1,5$ ), variando de 10 a 19 anos. Considerando a idade no primeiro parto, a diferença média entre o início da atividade sexual e o parto foi de 2,0 anos ( $\mathrm{DP}=1,4)$, variando de 0 (parto no mesmo ano do início de atividade sexual) até oito anos. A idade média do companheiro foi de 


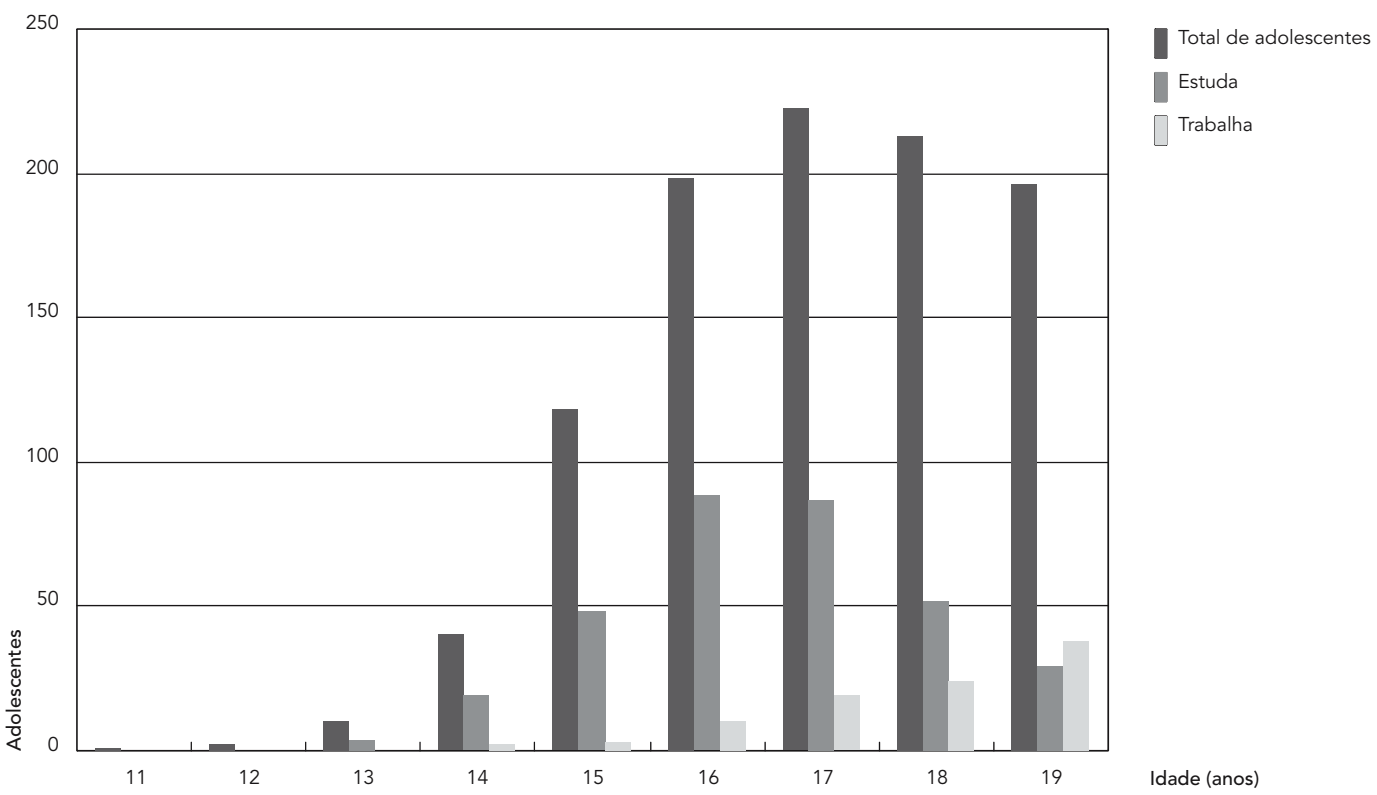

21 anos $(\mathrm{DP}=4,4)$, variando de 15 a 51 anos. A média de diferença de idade entre a adolescente e seu parceiro foi 4,0 anos ( $D P=4,3$ ) variando de menos 3 (parceiro mais novo) a 32 anos.

Para analisar melhor algumas características, foram elaboradas as Tabelas 2, 3 e 4, nas quais as adolescentes foram divididas em dois grupos: as mais novas (com 16 anos ou menos) e as demais (maiores que 16 anos). A Tabela 2 traz características do comportamento sexual. Não se observam diferenças significativas entre os grupos quanto à referência de que mantinham uma relação mutuamente fiel (87,9\% e 89,8\%) ou de que o comportamento sexual não sofreu mudanças em decorrência do advento da AIDS (51,8\% e 52,9\%), nem quanto à menção de nunca ou nem sempre usarem preservativo para prevenção de DST (80,3\% e 77,5\%). Apesar de nos dois grupos a maioria referir ter tido apenas um parceiro sexual $(66,7 \%$ e $49 \%)$, existe uma diferença significativa entre eles; além disso, é maior a porcentagem de adolescentes mais velhas que referem ter tido dois (15,3\% e $25 \%)$ e três ou mais (18\% e 26\%) parceiros na vida. Existem também diferenças significativas quanto ao tempo que mantêm uma relação mutuamente fiel. Para as mais novas, predomina o tempo de relações de 1 a 3 anos $(64,8 \%)$, seguido de até um ano $(21,8 \%)$, ao passo que, para as mais velhas, embora também predominem as relações de 1 a 3 anos, estas são seguidas das que duram mais de três anos. Quanto ao número de filhos vivos, nos dois grupos predomina a referência de que o concepto era o primeiro bebê $(89,1 \%$ e $72,6 \%)$, mas as porcentagens de dois $(17,1 \%)$ e três ou mais $(3,8 \%)$ das mais velhas são significativamente diferentes e superiores quando comparadas com as porcentagens das mais novas $(4,9 \%$ e $0,5 \%)$.

A idade média do filho mais velho era de 2,0 anos (DP $=1,27)$, variando de 1 a 8 anos. Em 147 casos $(91,3 \%)$, esses filhos moravam com a mãe; em $13(8,1 \%)$, eles moravam com parentes próximos, e um caso $(0,6 \%)$ foi colocado para adoção.

A Tabela 3 descreve características da gestação atual. A única diferença significativa encontrada entre os dois grupos foi o local do prénatal, predominando em $68,3 \%$ das mais novas o ambulatório do próprio hospital e em 52,4\% das mais velhas as unidades básicas de saúde. Para a maioria das entrevistadas, a gestação não 
Características da vida sexual de adolescentes grávidas internadas numa maternidade municipal de São Paulo, Brasil, segundo faixa de idade.

\begin{tabular}{|c|c|c|c|c|c|c|c|c|}
\hline \multirow[t]{2}{*}{ Características } & \multicolumn{2}{|c|}{ Total } & \multicolumn{2}{|c|}{$\leq 16$ anos } & \multicolumn{2}{|c|}{ Mais de 16 anos } & \multirow[t]{2}{*}{$\chi^{2}$} & \multirow[t]{2}{*}{ Valor $p$} \\
\hline & $\mathbf{N}$ & $\%$ & $\mathbf{N}$ & $\%$ & $\mathbf{N}$ & $\%$ & & \\
\hline Número de parceiros & & & & & & & 29,4 & $<0,001$ \\
\hline 1 & 552 & 55,5 & 244 & 66,7 & 308 & 49,0 & & \\
\hline 2 & 213 & 21,4 & 56 & 15,3 & 157 & 25,0 & & \\
\hline 3 ou + & 229 & 22,9 & 66 & 18,0 & 163 & 26,0 & & \\
\hline Relação mutuamente fiel & & & & & & & 0,835 & 0,2 \\
\hline Não & 107 & 10,7 & 44 & 12,1 & 63 & 10,2 & & \\
\hline Sim & 877 & 87,9 & 321 & 87,9 & 556 & 89,8 & & \\
\hline Tempo de relação fiel (anos) & & & & & & & 37,4 & $<0,002$ \\
\hline$\leq 1$ & 167 & 16,7 & 70 & 21,8 & 97 & 17,2 & & \\
\hline $1-3$ & 494 & 49,4 & 208 & 64,8 & 286 & 50,8 & & \\
\hline$>3$ & 223 & 22,3 & 43 & 13,4 & 180 & 32,0 & & \\
\hline Mudança de comportamento & & & & & & & 0,112 & 0,9 \\
\hline Nada & 524 & 52,4 & 191 & 51,8 & 333 & 52,9 & & \\
\hline Pouco & 187 & 18,7 & 70 & 19,0 & 117 & 18,6 & & \\
\hline Muito & 288 & 28,8 & 108 & 29,3 & 180 & 28,6 & & \\
\hline Uso de preservativo & & & & & & & 1,16 & 0,56 \\
\hline Nunca & 429 & 43,2 & 162 & 44,1 & 267 & 42,7 & & \\
\hline Nem sempre & 351 & 35,3 & 133 & 36,2 & 218 & 34,8 & & \\
\hline Sempre & 213 & 21,5 & 72 & 19,6 & 141 & 22,5 & & \\
\hline Número de filhos vivos & & & & & & & 45,1 & $<0,001$ \\
\hline Nenhum & 61 & 6,1 & 20 & 5,5 & 41 & 6,5 & & \\
\hline Atual & 784 & 78,4 & 326 & 89,1 & 458 & 72,6 & & \\
\hline 2 & 126 & 12,6 & 18 & 4,9 & 108 & 17,1 & & \\
\hline 3 ou + & 26 & 2,6 & 2 & 0,5 & 24 & 3,8 & & \\
\hline
\end{tabular}

foi planejada $(83,7 \%$ e $80 \%)$, mas foi considerada conveniente na ocasião do parto (58,2\% e 63,2\%); a minoria $(20,9 \%$ e $25,6 \%)$ fazia uso de algum método contraceptivo quando engravidou.

Pensaram interromper a gestação 127 adolescentes (12,7\%), e 15 destas realmente tentaram o aborto, a grande maioria referindo o uso de chás, mas em apenas oito houve realmente o aborto (cinco espontâneos e três provocados).

Quanto ao pré-natal, 92,7\% das mais novas e $90,3 \%$ das mais velhas referiram ter acompanhamento, comparecendo a seis ou mais consultas (65,3\% e $65,6 \%)$. Relataram faltas ao pré-natal $25,1 \%$ e $20,9 \%$ das adolescentes e o principal motivo alegado foi o esquecimento.

Quanto a hábitos e/ou uso de drogas durante a gestação, 173 adolescentes (17,3\%) referiram fumar em média cinco cigarros por dia ( $\mathrm{DP}=7,2)$, variando de 1 a 50 cigarros/dia. Quanto à ingestão de álcool, 266 adolescentes (26,6\%) admitiram ter ingerido pelo menos em uma ocasião durante a gestação, sendo 28 (2,8\%) de forma abusiva. No que tange a outros tipos de drogas (maconha, cocaína), 17 (1,7\%) admitiram ter usado durante a gestação e seis $(0,6 \%)$ destas relataram uso de droga injetável. Referiram ter tido relação com um parceiro usuário de droga injetável 24 (2,4\%) adolescentes.

Na Tabela 4, são apresentados alguns resultados perinatais. Na comparação dos dois grupos, a única diferença significativa encontrada foi o fato de ser a primeira gestação para $91,6 \%$ das mais novas e $73,3 \%$ das mais velhas. Nas demais comparações, observaram-se algumas diferenças entre os grupos, mas não foram estatisticamente significativas. Deram à luz um bebê vivo $94,3 \%$ e $91,1 \%$; o tipo de parto foi vaginal em $69,8 \%$ e $65 \%$ das adolescentes. Quanto aos bebês nascidos vivos, $14,4 \%$ e $12,6 \%$ foram pré-termo; $17,2 \%$ e $15 \%$ pesaram menos de $2.500 \mathrm{~g}$, ressaltando-se que, apesar de as diferenças entre os grupos não serem significativas, as porcentagens mostram uma tendência de os índices de prematuridade e baixo peso serem superiores nas mais novas. 
Características da gestação atual de adolescentes grávidas internadas numa maternidade municipal de São Paulo, Brasil, por faixa de idade.

\begin{tabular}{|c|c|c|c|c|c|c|c|c|}
\hline \multirow[t]{2}{*}{ Características } & \multicolumn{2}{|c|}{ Total } & \multicolumn{2}{|c|}{$\leq 16$ anos } & \multicolumn{2}{|c|}{ Mais de 16 anos } & \multirow[t]{2}{*}{$\chi^{2}$} & \multirow[t]{2}{*}{ Valor $p$} \\
\hline & $\mathbf{N}$ & $\%$ & $\mathbf{N}$ & $\%$ & $\mathbf{N}$ & $\%$ & & \\
\hline Gestação planejada & & & & & & & 2,18 & 0,81 \\
\hline Não & 812 & 81,2 & 309 & 83,7 & 503 & 80,0 & & \\
\hline Sim & 186 & 18,6 & 60 & 16,3 & 126 & 20,0 & & \\
\hline Época em que ocorreu & & & & & & & 2,38 & 0,07 \\
\hline Inconveniente & 382 & 38,6 & 152 & 41,8 & 230 & 36,8 & & \\
\hline Conveniente & 607 & 61,4 & 212 & 58,2 & 395 & 63,2 & & \\
\hline \multicolumn{9}{|l|}{ Uso de método anticoncepcional } \\
\hline quando engravidou & & & & & & & 2,70 & 0,057 \\
\hline Não & 757 & 76,2 & 291 & 79,1 & 466 & 74,4 & & \\
\hline Sim & 237 & 23,8 & 77 & 20,9 & 160 & 25,6 & & \\
\hline Refere pré-natal & & & & & & & 1,60 & 0,12 \\
\hline Não & 88 & 8,8 & 27 & 7,3 & 61 & 9,7 & & \\
\hline Sim & 909 & 91,2 & 341 & 92,7 & 568 & 90,3 & & \\
\hline Local do pré-natal & & & & & & & 53,77 & $<0,001$ \\
\hline Maternidade & 480 & 52,7 & 233 & 68,3 & 247 & 43,4 & & \\
\hline Unidade básica de saúde & 395 & 43,4 & 97 & 28,4 & 298 & 52,4 & & \\
\hline Outros & 35 & 3,8 & 11 & 3,2 & 24 & 4,2 & & \\
\hline Consultas de pré-natal referidas & & & & & & & 0,00 & 0,49 \\
\hline $1-5$ & 293 & 34,5 & 109 & 34,7 & 184 & 34,4 & & \\
\hline 6 ou + & 556 & 65,5 & 205 & 65,3 & 351 & 65,6 & & \\
\hline Falta ao pré-natal & & & & & & & 2,14 & 0,08 \\
\hline Não & 701 & 77,5 & 254 & 74,9 & 447 & 79,1 & & \\
\hline Sim & 203 & 22,5 & 85 & 25,1 & 118 & 20,9 & & \\
\hline
\end{tabular}

Tabela 4

Resultados perinatais de adolescentes grávidas internadas numa maternidade municipal de São Paulo, Brasil, segundo faixa de idade.

\begin{tabular}{|c|c|c|c|c|c|c|c|c|}
\hline \multirow[t]{2}{*}{ Características } & \multicolumn{2}{|c|}{ Total } & \multicolumn{2}{|c|}{$\leq 16$ anos } & \multicolumn{2}{|c|}{ Mais de 16 anos } & \multirow[t]{2}{*}{$\chi^{2}$} & \multirow[t]{2}{*}{ Valor $p$} \\
\hline & $\mathbf{N}$ & $\%$ & $\mathbf{N}$ & $\%$ & $\mathbf{N}$ & $\%$ & & \\
\hline Teve um bebê & & & & & & & 3,350 & $<0,42$ \\
\hline Não & 77 & 7,7 & 21 & 5,7 & 56 & 8,9 & & \\
\hline Sim & 922 & 92,3 & 348 & 94,3 & 574 & 91,1 & & \\
\hline Primeira gestação & & & & & & & 48,669 & $<0,001$ \\
\hline Não & 199 & 19,9 & 31 & 8,4 & 168 & 26,7 & & \\
\hline Sim & 800 & 80,1 & 338 & 91,6 & 462 & 73,3 & & \\
\hline Tipo de parto & & & & & & & 2,270 & 0,322 \\
\hline Vaginal & 668 & 67,3 & 257 & 69,8 & 411 & 65,8 & & \\
\hline Cesárea & 256 & 25,8 & 90 & 24,5 & 69 & 6,9 & & \\
\hline Curetagem pós-abortamento & 69 & 6,9 & 21 & 5,7 & 48 & 7,7 & & \\
\hline Peso do recém-nascido & & & & & & & 1,720 & 0,42 \\
\hline Normal & 774 & 84,1 & 287 & 82,7 & 487 & 85,0 & & \\
\hline Baixo peso & 124 & 13,5 & 49 & 14 & 75 & 13,1 & & \\
\hline Muito baixo peso & 22 & 2,4 & 11 & 3,2 & 11 & 1,9 & & \\
\hline Idade gestacional & & & & & & & 0,639 & 0,24 \\
\hline Pré-termo & 122 & 13,3 & 50 & 14,4 & 72 & 12,6 & & \\
\hline Termo & 798 & 86,7 & 297 & 85,6 & 501 & 87,4 & & \\
\hline
\end{tabular}


Pretendiam amamentar o bebê 905 adolescentes (90,9\%). Quanto aos pais dos bebês, 975 $(98,2 \%)$ sabiam da gestação e $799(80,6 \%)$ já tinham conhecimento donascimentonaocasiãoda entrevista. Entre esses, a maioria $(70,7 \%)$ é mencionada como tendo ficado feliz com a gestação.

Em relação a situações de violência referidas pela própria adolescente, $81(8,1 \%)$ já haviam sido atacadas com uma arma e 80 (8\%), sem arma. Já haviam sofrido ameaças de violência em geral $104(10,4 \%)$ adolescentes e $51(5,1 \%)$ haviam sofrido violência sexual. Em 24 situações de violência doméstica, foi necessário solicitar ajuda policial; dentre estas, 21 ocorreram durante a atual gestação.

\section{Discussão}

Os dados selecionados do projeto temático Uso de Drogas em Gestantes Adolescentes e apresentados neste artigo permitem caracterizar, sócio-demográfica e comportamentalmente, as adolescentes pertencentes à região Norte da periferia de um grande centro urbano como é o Município de São Paulo. Essa região, na análise da Fundação Seade 19, inclui distritos (Brasilândia e Cachoeirinha ) com os maiores índices de vulnerabilidade juvenil do município.

Durante o período do estudo, a alta taxa de adolescentes grávidas internadas $(24,3 \%)$, sendo $17 \%$ com até 15 anos, é condizente com os dados apresentados pelo Ministério da Saúde 14 e por outros estudos brasileiros 15,16,17,18. Esses estudos destacam a alta e crescente taxa de gravidez na adolescência, principalmente entre as mais novas, e confirmam que este é um fenômeno que tem de ser encarado como um problema sério de saúde pública. Trata-se de um fenômeno complexo, com manifestações específicas em cada região do país $15,17,18,23$. No Município de São Paulo, no boletim da Secretaria Municipal de Saúde 24, a proporção de recém-nascidos vivos de mães com menos de vinte anos foi de 16,6\% em 2001 e $16 \%$ em 2002. A taxa de $24,3 \%$ encontrada no presente estudo reflete que o parto de mães adolescentes da região Norte da cidade se concentra neste hospital.

Os principais aspectos desta pesquisa foram o tamanho da amostra, com perda praticamente nula, e a importância do tema. Como limitação, apontamos o fato de não se terem acompanhado longitudinalmente as adolescentes e de ter-se realizado o questionário com a jovem ainda no hospital, logo após o parto ou a curetagem.

$\mathrm{O}$ pequeno índice de casamentos formais $(7,2 \%)$ e o grande número de uniões consensuais $(60,6 \%)$ em conseqüência da gravidez demons- tram que, nessa classe social, existe uma pressão social para que o casal formalize uma união e passe a conviver sob o mesmo teto, mesmo sem oficializar o casamento ou ter uma independência financeira. O número de pessoas que convivem na mesma casa e o fato de a maioria residir em casa própria revelam a maneira como as famílias na periferia assimilam novas uniões motivadas pela gravidez precoce. Mãe, bebê e muitas vezes também o companheiro passam a morar com as famílias de origem, tendo-se, assim, vários núcleos familiares convivendo num mesmo espaço físico, compondo e dividindo a renda e a organização familiar.

Um importante aspecto levantado se refere à escolaridade das adolescentes. A proporção de jovens de 15 a 17 anos que não freqüentam a escola segundo a Fundação Seade 19 gira em torno de 25\% para o Município de São Paulo. Neste estudo, porém, o índice de evasão escolar observado $(67,3 \%)$ foi muito mais elevado. A evasão associada à gestação precoce traz graves conseqüências para a adolescente e seu filho e para a sociedade em geral, principalmente porque, nessa faixa etária, uma das poucas opções de inserção social e de ascensão econômica se dá por intermédio do sistema educacional. A relação entre gravidez precoce e suas repercussões na escolaridade tem sido destacada na literatura especializada 11, e experiências internacionais relatam programas preventivos desenvolvidos com base nesse tema, como a apresentada por Barnet et al. ${ }^{11}$ para a cidade de Baltimore.

O início precoce da atividade sexual e, principalmente, de forma desprotegida, associado com o alto índice de gestações não planejadas decorrentes de relacionamento com parceiro igualmente jovem são dados que desencadeiam reflexões sobre nossos adolescentes, que, apesar de razoável nível de escolaridade e de conhecimento sobre sexualidade, não conseguem traduzi-los em sexo protegido e mudanças de comportamento. A repetição de nova gestação indesejada ainda na adolescência de uma em cada cinco jovens reflete que nem a vivência da gestação e suas conseqüências são efetivas para o desenvolvimento de um comportamento sexual responsável, capaz de romper um círculo vicioso. Pesquisas de abordagem predominantemente qualitativa 25,26 têm enriquecido estas reflexões, contribuindo com a compreensão de que, em determinadas situações, a gravidez precoce possa ser a solução para situações conflituosas e não necessariamente um problema em si. Isso reflete a complexidade do fenômeno denominado "gravidez precoce".

Em relação aos resultados perinatais, estudos brasileiros 27,28 falam da associação de prematu- 
ridade e baixo peso ao nascer com idade materna, condições sócio-econômicas desfavoráveis, assistência pré-natal pública e tipo de parto. Os elevados índices de prematuridade $(13,3 \%)$ e de baixo peso $(15,9 \%)$ ao nascer encontrados no presente estudo confirmam essa associação, pois a população estudada era de mães adolescentes, predominantemente de classes sociais desfavorecidas, com assistência pré-natal no SUS. Esses dados fornecem subsídios para a elaboração de políticas de saúde e devem ser considerados nas prioridades da atenção materno-infantil.

A associação de gravidez precoce a outros comportamentos de risco, como o uso e abuso de drogas numa população jovem, inserida num contexto de situações de violência, caracteriza a população estudada como extremamente vulnerável, que necessita de abordagens específicas e urgentes se desejarmos mudar o panorama encontrado.

Resumindo, conforme já apontado por Singh 10 e confirmado pelo presente estudo, a gestação na adolescência é um fenômeno com repercussões significativas para o indivíduo e para a sociedade. Para a adolescente, a gravidez precoce pode marcar e alterar toda a sua vida. Pela perspectiva da comunidade e do governo, esse fenômeno tem uma forte associação com baixos níveis educacionais e um impacto negativo no seu potencial de ascensão econômica.

\section{Conclusão}

A gravidez na adolescência é um fenômeno complexo, associado a grande número de fatores, como os econômicos, educacionais e comportamentais, precipitando problemas e desvantagens decorrentes da maternidade precoce.

O presente estudo fornece subsídios importantes para o estabelecimento de estratégias específicas e políticas públicas preventivas visando à redução de comportamentos de risco.

\section{Resumo}

Com objetivo de identificar o perfil sócio-demográfico e comportamental de gestantes adolescentes, foram entrevistadas mil adolescentes, admitidas entre 24 de julho de 2001 e 27 de novembro de 2002, em um hospital municipal de São Paulo, Brasil, correspondendo a $24,3 \%$ das internações para resolução da gestação. Setenta (7\%) adolescentes foram admitidas para curetagem pós-abortamento e 930 (93\%), para parturição, com idade média de $17 \pm 1,5$ anos. A maioria $(72,4 \%)$ residia próximo ao hospital e $93 \%$ pertenciam às classes econômicas $C, D$ e E. Na época da parturição, 627 (67,3\%) adolescentes não estudavam. Apenas 23,7\% das adolescentes faziam uso de método contraceptivo, 81,2\% não tinham planejado a gestação e $80,1 \%$ eram primigestas. Dos partos, $67,4 \%$ foram vaginais. Dos bebês, 13,3\% foram pré-termo e 15, 9\%, baixo peso. Durante a gestação, o consumo referido de tabaco, de álcool e de drogas ilícitas foi, respectivamente de 17,3\%, 2,8\% e 1,7\%. Conclui-se que gravidez na adolescência é um fenômeno complexo, associado a fatores econômicos, educacionais e comportamentais, precipitando problemas decorrentes da maternidade precoce. O presente estudo fornece subsídios para políticas públicas de saúde, visando a prevenir a gravidez na adolescência.

Gravidez na Adolescência; Adolescente; Classe Social; Demografia; Comportamento Sexual

\section{Colaboradores}

E. Chalem, S. S. Mitsuhiro e R. Laranjeira participaram da concepção e elaboração do estudo, da coleta de dados, da análise e interpretação dos dados e da elaboração e revisão crítica do artigo. C. P. Ferri participou da análise e interpretação dos dados e da elaboração e revisão crítica do artigo. M. C. M. Barros e R. Guinsburg participaram da concepção e elaboração do estudo, da coleta de dados e da revisão crítica do artigo.

\section{Agradecimentos}

O projeto temático Uso de Drogas em Gestantes Adolescentes foi financiado pela Fundação de Amparo à Pesquisa do Estado de São Paulo (processo 00/10.293-5). 


\section{Referências}

1. Cunnnington AJ. What's so bad about teenage pregnancy? J Fam Plann Reprod Health Care 2001; 27:36-41.

2. Furstenberg Jr. FF, Brooks-Gunn J, Chase-Lansdale. Am Psychol 1989; 44:313-20.

3. Goodyear RK, Newcomb MD, Locke TF. Pregnant Latin teenagers: psychosocial and developmental determinants of how they select and perceive the men who father their children. J Couns Psychol 2002; 49:187-201.

4. Lawlor DA, Shaw M. Teenage pregnancy rates: high compared with where and when? J R Soc Med 2004: 97:121-3.

5. Marecek J. Counseling adolescents with problem pregnancies. Am Psychol 1987; 42:89-93.

6. Senanayake P, Faulkner KM. Unplanned teenage pregnancy. Best Pract Res Clin Obstet Gynaecol 2003; 17:117-29.

7. Mayor S. Pregnancy and childbirth are leading causes of death in teenage girls in developing countries. BMJ 2004; 328:1152.

8. Henshaw SK. Teenage abortion and pregnancy statistics by state, 1992. Fam Plann Perspect 1997; 29:115-22.

9. Bennett T, Skatrud JD, Guild P, Loda F, Klerman LV. Rural adolescent pregnancy: a view from the South. Fam Plann Perspect 1997; 29:256-67.

10. Sing S. Adolescent childbearing in developing countries: a global review. Stud Fam Plann 1998; 29:117-36.

11. Barnet B, Arroyo C, Devoe M, Duggan AK. Reduced school dropout rates among adolescent mothers receiving school-based prenatal care. Arch Pediatr Adolesc Med 2004; 158:262-8.

12. Arias E, MacDorman MF, Strobino DM, Guyer B. Annual summary of vital statistics - 2002. Pediatrics 2003; 112:12151-230.

13. Creatsas GC. Adolescent pregnancy in Europe. Int J Fertil Menopausal Stud 1995; 40 Suppl 2:80-4.

14. Ministério da Saúde. Gravidez na adolescência. http://portal.saude.gov.br/saude/visao.cfm?id (acessado em 27/Fev/2004)

15. Gama SGN, Szwarcwald CL, Leal MC. Experiência de gravidez na adolescência, fatores associados e resultados perinatais entre puérperas de baixa renda. Cad Saúde Pública 2002; 18:153-61.

16. Sabroza AR, Leal MC, Gama SGN, Costa JV. Perfil sócio-demográfico e psicossocial de puérperas adolescentes do Município do Rio de Janeiro, Brasil - 1999-2001. Cad Saúde Pública 2004; 20 Suppl 1:S112-20.

17. Ribeiro ER, Barbieri MA, Bettiol H, Silva AA. Comparação entre duas coortes de mães adolescentes em município do Sudeste do Brasil. Rev Saúde Pública 2000; 34:136-42.
18. Simões VM, Silva AA, Bettiol H, Lamy-Filho F, Tonial SR, Mochel EG. Características da gravidez na adolescência em São Luís, Maranhão. Rev Saúde Pública 2003; 37:559-65.

19. Fundação Sistema Estadual de Análise de Dados. Índice de vulnerabilidade juvenil. http://www. seade.gov.br/ivj/ (acessado em 04/Dez/2004).

20. Galduroz JCF, Noto AR, Carlini EA. IV levantamento sobre o uso de drogas entre estudantes de 1 o e 2 o graus em 10 capitais brasileiras. São Paulo: Centro Brasileiro de Informações sobre Drogas Psicotrópicas, Departamento de Psicobiologia, Escola Paulista de Medicina; 1997.

21. Zahnd E, Klein D, Needell B. Substance use and issues of violence among low-income, pregnant women: The California Perinatal Needs Assessment. J Drug Issues 1997; 27:563-84.

22. Associação Nacional de Empresas de Pesquisa. Critério de classificação econômica Brasil. São Paulo: Associação Nacional de Empresas de Pesquisa; 1997.

23. Aquino EML, Heilborn ML, Knauth D, Bozon M, Almeida MC, Araújo J, et al. Adolescência e reprodução no Brasil: a heterogeneidade dos perfis sociais. Cad Saúde Pública 2003; 19 Suppl 2: S377-88.

24. Secretaria Municipal de Saúde. Boletim Nascidos Vivos - CEInfo. Ano 1, n. 1; 2004. http://ww2.prefeitura.sp.gov.br//arquivos/secretarias/saude/ publicacoes/0004/BoletimSINASC.pdf (acessado em 10/Abr/2006).

25. Taquette SR. Sexo e gravidez na adolescência: estudo de antecedentes biopsicossociais [Dissertação de Mestrado]. Ribeirão Preto: Faculdade de Medicina de Ribeirão Preto, Universidade de São Paulo; 1991.

26. Taquete SR. Iniciação sexual da adolescente: o desejo, o afeto e as normas sociais [Tese de Doutorado]. Ribeirão Preto: Faculdade de Medicina de Ribeirão Preto, Universidade de São Paulo; 1997.

27. Bettiol H, Barbieri MA, Gomes UA, Wen LY, Reis PM, Chiaratti TM, et al. Atenção médica à gestação e ao parto de mães adolescentes. Cad Saúde Pública 1992; 8:404-13.

28. Bettiol H, Rona RJ, Chinn S, Goldani M, Barbieri MA. Factors associated with preterm births in southeast Brazil: a comparison of two birth cohorts born 15 years apart. Paediatr Perinat Epidemiol 2000; 14:30-8.

Recebido em 04/Jul/2005

Versão final reapresentada em 02/Mai/2006

Aprovado em 18/Mai/2006 